\title{
V. The scattering and regular reflexion of light by gas molecules.-Part II
}

\section{C.V. Burton D.Sc.}

To cite this article: C.V. Burton D.Sc. (1915) V. The scattering and regular reflexion of light by gas molecules.-Part II, Philosophical Magazine Series 6, 30:175, 87-105, DOI: $10.1080 / 14786440708635374$

To link to this article: http://dx.doi.org/10.1080/14786440708635374

曲 Published online: 08 Apr 2009.

Submit your article to this journal $₫$

Џ Article views: 3

Q View related articles $\square$

Citing articles: 1 View citing articles $\square$ 
V. The Scattering and Regular Reflexion of Light by Gas Molecules.-Part II. By C. V. Burron, D.Sc.*

28. TT will now be convenient to consider further a result 1 obtained in Part I.t The region $0<x<\mathrm{L}$ is occupied by a cloud of Rayleigh resonators (or more generally secondary vibrators) and plane sound-waves specified by the velocity-potential

$$
\psi=A \exp i(p t-v x)
$$

are incident normally on this region. A complex constant $\chi$ characteristic of the swarm is defined by the condition that if the resultant regular disturbance at the plane $x^{\prime}$ is $\mathrm{E}^{\prime}$ exp $i p t$, then the secondary plane-waves emitted by the elementary lamina $d x^{\prime}$ are

$$
-\chi \mathrm{E}^{\prime} \exp i\left\{p t \mp v\left(x-x^{\prime}\right)\right\} d x^{\prime} ;
$$

$\mathrm{B}^{\prime}$, or $-\chi \mathrm{E}^{\prime}$, as a function of $x^{\prime}$, being given by

$$
\mathrm{B}^{\prime}=\mathrm{C}_{1} \exp i \mu \xi+\mathrm{C}_{2} \exp (-i \mu \xi),
$$

where $\quad \mu \equiv \mathrm{N}\left(1-2 i \chi^{-1}\right), \quad \xi \equiv v x \equiv 2 \pi x / \lambda$,

and the constants $\mathrm{C}_{1}, \mathrm{C}_{2}$ are determined by (21), (22); which may be written

$$
\begin{aligned}
& 2 \chi \mathrm{A}=\mathrm{C}_{1}[-(\mu+1) \exp i(\mu-1) \eta+(\mu-1)] \\
& \quad+\mathrm{C}_{2}[(\mu-1) \exp \{-i(\mu+1) \eta\}-(\mu+1)] \\
& \begin{aligned}
2 \chi \mathrm{A}=\mathrm{C}_{1}[- & (\mu+1) \exp i(\mu+1) \eta+(\mu-1)] \\
& +\mathrm{C}_{2}[(\mu-1) \exp \{-i(\mu-1) \eta\}-(\mu+1)] .
\end{aligned}
\end{aligned}
$$

29. In the most general case (cf. $\S 21$ ) we may write

$$
\chi \equiv \chi_{1}+i \chi_{2}, \quad \mu \equiv \mu_{1}-i \mu_{2}, \quad . \quad \text {. . . }
$$

where $\chi_{1}, \chi_{2}, \mu_{1}, \mu_{2}$ are all real. Then from (19) $\mu_{1}, \mu_{2}$ may be found in terms of $\chi_{1}, \chi_{2}$; the positive sign being conveniently retained where a choice of signs is open. The results obtained are

$$
\begin{aligned}
& \mu_{1}=\sqrt{ }\left\{\frac{1}{2}\left(1+2 \chi_{2} v^{-1}\right)+\frac{1}{2} \sqrt{ }\left[\left(1+2 \chi_{2} v^{-1}\right)^{2}+4 \chi_{1}{ }^{2} v^{-2}\right]^{\prime}\right\}, \\
& \mu_{2}=\sqrt{ }\left\{-\frac{1}{2}\left(1+2 \chi_{2} v^{-1}\right)+\frac{1}{2} \sqrt{ }\left[\left(1+2 \chi_{2} v^{-1}\right)^{2}+4 \chi_{1}{ }^{2} v^{-2}\right]\right\} ;
\end{aligned}
$$

and evidently both $\mu_{1}$ and $\mu_{2}$ are always positive.

$$
\begin{aligned}
& \text { * Communicated by the Author. } \\
& \dagger \text { May } 1915 \text {. }
\end{aligned}
$$


Thus exp $i \mu \eta$, containing the real exponential factor $\exp \mu_{2} \eta$, becomes infinite with $\eta$, and at the same time $\exp (-i \mu \eta)$ vanishes. Accordingly, when the laminar region filled with secondary vibrators is infinitely thick, (25), (26) both lead to

$$
\mathrm{C}_{1}=0, \quad \mathrm{C}_{2}=-2 \chi \mathrm{A} /(\mu+1) ;
$$

and hence (with the accents dropped)

$$
\begin{aligned}
\mathrm{E}=-x^{-1} \mathrm{~B} & =2 \mathrm{~A}(\mu+1)^{-1} \exp (-i \mu v x) \\
& =2 \mathrm{~A}\left\{(\mu+1)^{2}+\mu_{2}^{2}\right\}^{-1}\left(\mu_{1}+1+i \mu_{2}\right) \exp \left(-\mu_{2} v x\right) \exp \left(-i \mu_{1} v x\right) \\
& =2 \mathrm{~A}\left\{\left(\mu_{1}+1\right)^{2}+\mu_{2}^{2}\right\}^{-\frac{1}{2}} \exp \left(-\mu_{2} v x\right) \exp i\left(-\mu_{1} v x+\zeta\right) ; \quad .
\end{aligned}
$$

where $\quad \cos \zeta, \sin \zeta \equiv \frac{\mu_{1}+1, \mu_{2}}{\left\{\left(\mu_{1}+1\right)^{2}+\mu_{2}^{2}\right\}^{\frac{1}{2}}} \quad$. . .

30. Analytically E expipt represents a train of waves propagated exclusively in the direction of $x$-increasing, the amplitude falling off in the proportion $e^{-1}$ for a distance traversed equal to $\lambda / 2 \pi \mu_{2}$, while the wave-velocity is $\mu_{1}^{-1}$ times what it would be if the secondary vibrators were absent. The refractive index in the ordinary optical sense is $\mu_{1}$, though the changed velocity and gradual extinction of the waves are both algebraically represented by the complex refractive index $\mu$.

31. The velocity-potential in the regularly reflected beam will evidently be of the form

$$
\psi^{\prime \prime \prime}=\mathrm{A}^{\prime \prime \prime} \exp i(p t+v x),
$$

where $A^{\prime \prime \prime}$ is a complex constant, determined by the condition that $A+A^{\prime \prime \prime}$ must be equal to the value assumed by $\mathrm{E}$ when $x=0$; that is

$$
\mathrm{A}^{\prime \prime \prime}=-\mathrm{A}+2 \mathrm{~A}\left\{\left(\mu_{1}+1\right)^{2}+\mu_{2}^{2}\right\}^{-1}\left(\mu_{1}+1+i \mu_{2}\right) .
$$

In order to trace more easily the strengthening of the reflected beam as the density of the swarm of vibrators is incrensed, consider the case where $\chi$ is real, and therefore identical with $\chi_{1}$, while $\chi_{2}$ vanishes. Then (28), (29) become

$(\chi \mathrm{real})$

$$
\mu_{1}=\sqrt{ }\left\{\frac{1}{2}+\frac{1}{2} \sqrt{ }\left[1+4 \chi^{2} v^{-2}\right]\right\}, . . .
$$

$$
\mu_{2}=\sqrt{ }\left\{-\frac{1}{2}+\frac{1}{2} \sqrt{ }\left[1+4 \chi^{2} v^{-2}\right]\right\} . . .
$$

First, when $\chi^{v^{-1}}$ (or $\lambda \chi / 2 \pi$ ) is small, we have as far as the 
Regular Reflexion of Light by Gas Molecules.

second order of $\chi^{v^{-1}}$

$$
\mu_{1} \fallingdotseq 1+\frac{1}{2} \chi^{2} v^{-2}, \quad \mu_{2} \fallingdotseq \chi v^{-1} ;
$$

and, as far as the first order of $\chi v^{-1}$,

$$
\mathrm{A}^{\prime \prime \prime} \fallingdotseq-\mathrm{A}+\mathrm{A}\left(1+\frac{1}{2} i \chi v^{-1}\right) \fallingdotseq-\frac{1}{2} \mathrm{~A} i \chi^{v^{-1}} .
$$

On the other hand, when $\chi v^{-1}$ becomes large (a condition which does not necessarily result from a large value of $\nu$ if absorption is then present), $\mu_{1}$ and $\mu_{2}$ both approach the value $\chi^{\frac{1}{2}} v^{-\frac{1}{2}}$, and (32) becomes in the limit

$$
\mathrm{A}^{\prime \prime \prime}=-\mathrm{A} \text {; }
$$

which implies that the reflexion has become complete.

32. So long as the (aërial) socondary vibrators are not too closely crowded to allow of their being distributed like the molecules of a gas, and so long as absorption is absent, the relation between $\chi$ and $\nu$ (the number of vibrators per unit of volume) is given by (13); while in the special case of resonators $\gamma=\frac{1}{2} \pi$, so that $\chi$ is real as in the last paragraph, and the approximation (35) is equivalent to

$$
\mathrm{A}^{\prime \prime \prime} \fallingdotseq-\mathrm{A} i \pi \nu v^{-3} \fallingdotseq-\mathrm{A} i \nu \lambda^{3} / 8 \pi^{2} . . .
$$

This result is true only when $\chi^{v^{-1}}$ or $\nu \lambda^{3} / 4 \pi^{2}$ is small; moreover it applies only to the acoustical case considered. The corresponding electromagnetic (or optical) case is referred to in $\S 48$.

33. Some practical interest attaches to the transmission of normally incident plane-waves through a lamina of finite thickness occupied by a swarm of secondary vibrators; for it is on this that certain interferometer measurements of anomalous dispersion are based. From (18), (19) we obtain, with the help of $(21),(22)$,

$$
\begin{array}{r}
\mathbf{E}=-\chi^{-1} \mathrm{~B}=-\chi^{-1}\left[\mathrm{C}_{1} \exp i \mu \xi+\mathrm{C}_{2} \exp (-i \mu \xi)\right] \\
=-2 \mathrm{~A}\{[(\mu-1) \exp \{-i(\mu-1) \eta\}-(\mu+1)] \exp i \mu \xi
\end{array}
$$$$
-[(\mu-1) \exp \{-i((\mu+1) \eta\}-(\mu+1)] \exp i \mu \xi
$$

$$
\begin{aligned}
+[-(\mu+1) \exp i & (\mu-1) \eta+(\mu-1)] \exp (-i \mu \xi) \\
& -[-(\mu+1) \exp i(\mu+1) \eta+(\mu-1)] \exp (-i \mu \xi)\}
\end{aligned}
$$

$\div\left\{(\mu+1)^{2} \exp i(\mu-1) \eta+(\mu-1)^{2} \exp \{-i(\mu-1) \eta\}\right.$

$$
\left.-(\mu-1)^{2} \exp \{-i(\mu+1) \eta\}-(\mu+1)^{2} \exp i(\mu+1) \eta\right\},
$$


and when $\xi=\eta$ (that is, when $x=\mathrm{L}$ ) this becomes

$$
\begin{aligned}
\mathrm{E}_{\eta}= & -8 \mathrm{~A} i \mu \sin \eta\left[(\mu+1)^{2} \exp i(\mu-1) \eta+(\mu-1)^{2} \exp \{-i(\mu-1) \eta\}\right. \\
& \left.-(\mu-1)^{2} \exp \{-i(\mu+1) \eta\}-(\mu+1)^{2} \exp i(\mu+1) \eta\right]^{-1} \\
= & 4 \mathrm{~A}\left(\mu_{1}{ }^{2}+\mu_{2}{ }^{2}\right)^{\frac{1}{2}} \mathrm{~W}^{-\frac{1}{2}} \exp i(-\eta-\vartheta-\pi) \quad . . . . .
\end{aligned}
$$

(as we find from (27) after some reductions), where

$$
\begin{aligned}
& \mathrm{W}=\left(\mu_{1}{ }^{2}+\mu_{2}{ }^{2}+1+2 \mu_{1}\right)^{2} \exp 2 \mu_{2} \eta+\left(\mu_{1}{ }^{2}+\mu_{2}{ }^{2}+1-2 \mu_{1}\right)^{2} \exp \left(-2 \mu_{2} \eta\right) \\
& -2\left(\mu_{1}^{4}+\mu_{2}^{4}+1+2 \mu_{1}^{2} \mu_{2}^{2}-2 \mu_{1}^{2}-6 \mu_{2}^{2}\right) \cos 2 \mu_{1} \eta \\
& +8 \mu_{2}\left(\mu_{1}^{2}+\mu_{2}^{2}-1\right) \sin 2 \mu_{1} \eta \\
& \cos \varpi=W^{-\frac{1}{2}}\left[\operatorname { e x p } \mu _ { 2 } \eta \cdot \left\{\left(\mu_{1}^{2}-\mu_{2}^{2}+1+2 \mu_{1}\right) \cos \left(\mu_{1}-1\right) \eta\right.\right. \\
& \left.+\left(2 \mu_{1} \mu_{2}+2 \mu_{2}\right) \sin \left(\mu_{1}-1\right) \eta\right\} \\
& +\exp \left(-\mu_{2} \eta\right) \cdot\left\{-\left(\mu_{1}^{2}-\mu_{2}^{2}+1-2 \mu_{1}\right) \cos \left(\mu_{1}+1\right) \eta\right. \\
& \left.\left.+\left(2 \mu_{1} \mu_{2}-2 \mu_{2}\right) \sin \left(\mu_{1}+1\right) \eta\right\}\right] \\
& \sin \varpi=\mathrm{W}^{-\frac{1}{2}}\left[\operatorname { e x p } \mu _ { 2 } \eta \cdot \left\{\left(\mu_{1}^{2}-\mu_{2}^{2}+1+2 \mu_{1}\right) \sin \left(\mu_{1}-1\right) \eta\right.\right. \\
& \left.-\left(2 \mu_{1} \mu_{2}+2 \mu_{2}\right) \cos \left(\mu_{1}-1\right) \eta\right\} \\
& +\exp \left(-\mu_{2} \eta\right)\left\{\left(\mu_{1}^{2}-\mu_{2}{ }^{2}+1-2 \mu_{1}\right) \sin \left(\mu_{1}+1\right) \eta\right. \\
& \left.\left.+\left(2 \mu_{1} \mu_{2}-2 \mu_{2}\right) \cos \left(\mu_{1}+1\right) \eta\right\}\right] \text {; }
\end{aligned}
$$

$\cos \vartheta, \sin \vartheta=\left(\mu_{1}, \mu_{2}\right)\left(\mu_{1}^{2}+\mu_{2}^{2}\right)^{-\frac{1}{2}}$.

Since $\mu_{1}, \mu_{2}$ are given in terms of $\chi_{1}, \chi_{2}$ by (28), (29), $\mathrm{E}_{\eta}$ is known in terms of the complex constant $\chi$.

34. The transmitted regular disturbance is thus

$$
\psi^{\prime \prime}=4 \mathrm{~A}\left(\mu_{1}^{2}+\mu_{2}^{2}\right)^{\frac{1}{2}} \mathrm{~W}^{-\frac{1}{2}} \exp p^{2}(p t-v x-\vartheta-\varpi),
$$

which assumes a simpler form when $\chi v^{-1}$ is small. For in that case (28), (29) become

so that

$$
\mu_{1} \fallingdotseq 1+\chi_{2} v^{-1}, \quad \mu_{2} \fallingdotseq \chi_{1} v^{-1},
$$

$$
\begin{gathered}
\mathrm{W}-\frac{1}{2} \fallingdotseq \frac{1}{4}\left(1+\chi_{2} v^{-1}\right)^{-1} \exp \left(-\chi_{1} \mathrm{~L}\right) \\
\sin \varpi \sin \chi_{2} \mathrm{~L}-\mu_{2} / \mu_{1} \cdot \cos \chi_{2} \mathrm{~L} \fallingdotseq \sin \left(\chi_{2} \mathrm{~L}-\vartheta\right)
\end{gathered}
$$

and (38) becomes

$$
\psi^{\prime \prime} \fallingdotseq \mathrm{A} \exp \left(-\chi_{1} \mathrm{~L}\right) \exp i\left(p t-v x-\chi_{2} \mathrm{~L}\right) .
$$

The refractivity, in the ordinary optical sense, is tlius, to our order of approximation, $\chi_{2}$; though strictly speaking $\mu_{1}-1$ does not vanish with $\chi_{2} v^{-1}$ when terms in $\chi_{1}^{2} v^{-2}$ are retained.

35. When the secondary vibrators are Rayleigh resonators, distributed through the region $0<x<\mathrm{L}$ with the complete irregularity of gas-molecules, the values of $\chi_{1}, \chi_{2}$ are given 
by (13), and the refractivity (as far as the first order of $\left.\chi_{1} v^{-1}, \chi_{2} v^{-1}\right)$ is

$$
\chi_{2} v^{-1}=\pi \nu v^{-3} \sin 2 \gamma=\nu \lambda^{3} \sin 2 \gamma / 8 \pi^{2}, \quad . \quad .
$$

while the coefficient of extinction, to the same order of approximation, is

$$
\chi_{1}=2 \pi \nu v^{-2} \sin ^{2} \gamma=\nu \lambda^{2} \sin ^{2} \gamma / 2 \pi .
$$

36. Up to this point the relations obtained belong to two classes : those which are peculiar to the case of simple aërial vibrators excited by sound-waves, and those which are true for swarms of similar vibrators of any type excited by planewaves of corresponding type; this latter, more general, class comprising the results of $\$ \$ 17-22,28-31,33,34$. The special features of the optical (or electromagnetic) problem remain to be considered.

37. In dealing with the excitation of an electromagnetic secondary vibrator by plane-waves, we shall have to apply Poynting's theorem, involving the vector-product of two vectors, so that the use of imaginary exponents is no longer admissible : only real quantities must appear in our equations *. The state of things close to a radiating atom is as yet involved in obscurity, and it will be well to avoid all assumptions as to intra-atomic conditions, the activity of a radiating atom being specified by the expression for the disturbance which it produces at a distance. The typical radiator, situated at the origin, and vibrating periodically in. a mode symmetrical with respect to the axis of $z$, produces at distant points a variable vector-potential

$$
\mathbf{a}^{\prime}=0,0, \mathrm{C} r^{-1} \cos (p t-v r-\gamma) ; \quad . \quad .
$$

where as before $p / 2 \pi$ is the frequency and $2 \pi / v$ the wavelength, while $r$ is the distance from the vibrator to the point at which $\mathbf{a}^{\prime}$ is measured $\dagger$. For the magnetic vector $\mathbf{h}^{\prime}$

* In the simpler case of an aërial resonator, Lord Rayleigh avoids the necessity for evaluating the time-average of the product pressure $X$ surfice-integral-of-normal-velocity; instead he introduces the condition that the two factors of this product (each represented by a complex exponential) must be in quadrature. The present case is rather nore complicated, and a more laborious treatment seems to be required.

t The Heaviside system of units, as adopted by Lorentz and other authorities, is here used; the relations of the various electromagnetic quantities being as stated. It may be observed at once that our final equations, dealing as they do with relative amplitudes or energies of incident, transmitted, reflected, and scattered radiation, will remain unaffected by any change in the units adopted for electromaqnetic measurements. The only furmula required are those belonging to the free æther, for the influence of any matter which may be present takes the form of secondary radiations, which are assumed throughout to have the same frequency as the primary waves. 
therefore, when $r$ is large in comparison with the wave-length, we get

$$
\mathbf{h}^{\prime}=\operatorname{rot} \mathbf{a}^{\prime}=\mathrm{C} v r^{-2} \sin (p t-v r-\gamma)(y,-x, 0) ; .
$$

while if $\mathrm{d}^{\prime}$ is the electric vector, and $c$ the velocity of light,

$$
\dot{\mathbf{d}}^{\prime}=c \operatorname{rot} \mathbf{h}^{\prime}=\mathrm{C} p v r^{-3} \cos (p t-v r-\gamma)\left(-x z,-y z, x^{2}+y^{2}\right) \cdot
$$

Hence, since the disturbance which concerns us is periodic,

$$
\mathrm{d}^{\prime}=C \boldsymbol{v} r^{-3} \sin (p t-v r-\gamma)\left(-x z,-y z, x^{2}+y^{2}\right) .
$$

38. If the primary (plane-polarized) waves, which cause the secondary disturbance (42) to be emitted, are represented by

$$
\mathrm{a}=0,0, \mathrm{~A} \cos (p t-v x), . . .
$$

and if the phase-lag $\gamma$ is known, $\mathrm{C}$ can be found in terms of $A$. From (45), $h$ and $d$ for the primary waves are seen to be

$$
\begin{aligned}
& \mathbf{h}=0,-\mathbf{A} v \sin (p t-v x), 0 ; . . \\
& \mathbf{d}=0,0, \mathbf{A} v \sin (p t-v x) .
\end{aligned}
$$

We have now to introduce the condition that the secondary vibrator merely scatters energy of frequency $p / 2 \pi$, without changing its total amount. To do this, we may imagine a closed surface drawn enclosing the vibrator, and write down the expression for the surface-integral of the Poynting vector; the condition then is that the time-average of this surface-integral should vanish. More conveniently the closed surface is replaced by two parallel planes, $x=-l$ and $x=l$, where $l: \lambda$ is large. Thus of the vector-product of $\mathrm{d}+\mathrm{d}^{\prime}$ and $\mathrm{h}+\mathrm{h}^{\prime}$ there is only the $x$-component to be considered, namely

$$
\begin{array}{r}
v^{2}\left\{\mathrm{~A} \sin (p t-v x)+\mathrm{C} r^{-3}\left(x^{2}+y^{2}\right) \sin (p t-v r-\gamma)\right\}\{\mathrm{A} \sin (p t-v x) \\
\left.+\mathrm{C} r^{-2} x \sin (p t-v r-\gamma)\right\} \\
=v^{2}\left[\mathrm{~A}^{2} \sin ^{2}(p t-v x)+\mathrm{C}^{2} r^{-5} x\left(x^{2}+y^{2}\right) \sin ^{2}(p t-v r-\gamma)\right. \\
\left.+\mathrm{AC}\left\{r^{-3}\left(x^{2}+y^{2}\right)+r^{-2} x\right\} \sin (p t-v x) \sin (p t-v r-\gamma)\right] .
\end{array}
$$

The quantity which has to vanish is the surface-integral of the last-written expression over the plane $x=l$ minus the surface-integral over the plane $x=-l$. Accordingly those terms may be omitted from the expression which remain unaltered when the sign of $x$ is reversed. On averaging the remaining terms with respect to time, and taking account of 
both planes, we get as the multiplier of $d y d z$ in the final integral

$$
\begin{aligned}
& v^{2}\left|\frac{1}{2} \mathrm{C}^{2} r^{-5} x\left(x^{2}+y^{2}\right)+\frac{1}{2} \mathrm{AC}\left\{r^{-3}\left(x^{2}+y^{2}\right)+r^{-2} x\right\} \cos \{v(r-x)+\gamma\}\right|_{x=-l}^{x=l} \\
& =v^{2}\left[\mathrm{C}^{2} r^{-5} l\left(l^{2}+y^{2}\right)+\frac{1}{2} \mathrm{AC} r^{-2} l\{\cos (v \cdot \overline{r-l}+\gamma)+\cos (v \cdot \overline{r+l}+\gamma)\}\right. \\
& \quad+\frac{1}{2} \mathrm{AC} r^{-3}\left(l^{2}+y^{2}\right)\{\cos (v \cdot r-l+\gamma)-\cos (v \cdot \overline{r+l}+\gamma)\}
\end{aligned}
$$

Since $l$ is arbitrary, let it be so chosen that $v l$ is a multiple of $2 \pi ; l$ may then be omitted from the arguments of the cosines, and dropping also the constant factor $\mathrm{C} v^{2} l$, we have for the quantity whose surface-integral must vanish

$$
\mathrm{C} r^{-5}\left(l^{2}+y^{2}\right)+\mathrm{A} r^{-2} \cos (\boldsymbol{v} r+\gamma) .
$$

39. If $q^{2} \equiv y^{2}+z^{2}$, the above expression must be multiplied by $2 \pi q d q$, that is by $2 \pi r d r$, and integrated from $r=l$ to $r=\infty$, the result being equated to zero. Thus

$\mathrm{C} \int_{l}^{\infty} r^{-4}\left(l^{2}+y^{2}\right) d r+\mathrm{A} \int_{l}^{\infty} r^{-1}(\cos v r \cos \gamma-\sin v r \sin \gamma) d r=0$

In the first integral, for any given value of $r, y^{2}$ may be replaced by its average value $\frac{1}{2} q^{2}=\frac{1}{2}\left(r^{2}-l^{2}\right)$. The first term in (48) is therefore

$$
\frac{1}{2} \mathrm{C}\left|-\frac{1}{3} l^{2} r^{-3}-r^{-1}\right|_{r=l}^{r=\infty}=\frac{2}{3} \mathrm{C} l^{-1} .
$$

The second term in (48) may be dealt with in two portions, each of which, by successive integration by parts, is transformed into a series of descending powers of $v l$. Remembering that $v l$ may be chosen as large as we please, and that it has already been designated a multiple of $2 \pi$, we readily obtain for the second integral the value $-A v^{-1} l^{-1} \sin \gamma$. Hence finally $\mathrm{C}=\frac{3}{2} \mathrm{~A} v^{-1} \sin \gamma$, and (42) becomes

[Type I.] $\quad \mathbf{a}^{\prime}=0,0, \frac{3}{2} \frac{\mathrm{A}}{v r} \sin \gamma \cos (p t-v r-\gamma) ; .$.

corresponding to the primary disturbance (45).

40. By way of example, two types of electromagnetic vibrators are considered in this paper; type I., whatever its orientation, when excited by plane-polarized waves is capable of vibrating symmetrically with respect to an axis parallel to the electric vector in those waves. Such a vibrator, placed at the origin and excited by primary waves (45), emits the secondary disturbance (49) in which the 
phase-lag $\gamma$ depends; on the "tuning"; the response being greatest when $\gamma=\frac{\pi}{2}$, that is, whence there is resonance. 'l'he vibrator or radiator of type II. is specified in $\S 42$.

41. Consider next a multitude of vibrators of type I., all lying in the plane of $y z$, and within a square whose sides are formed by the lines $y=\mp b, z=\mp b$. If these vibrators are distributed with complete irregularity, and are all vibrating. with the same amplitude and in the same phase, the vibrations of each being symmetrical about an axis parallel to the axis of $z$, the method of $\S \S 4-7$ can be applied to determine the ratio of the plane-wave energy propagated (say) in the direction of $x$-decreasing to the energy irregularly scattered. The notation need not be changed, if $a$ is now understood to be the amplitude of the electric vector due to a single vibrator at a point distant $f$ from it, the direction of $f$ being perpendicular to the $z$-axis. The expression $\sigma^{2} b^{2} a^{2} \lambda^{2} f^{2}$ represents as before, on an arbitrary scale, the energy-flux in the diffraction pattern ; while the average value of $\sin ^{2} \theta$ enters as a new factor into the corresponding expression $4 \pi \sigma b^{2} a^{2} f^{2} a v \cdot \sin ^{2} \theta$ for the scattered energy ; $\theta$ being the co-latitude of any point on a complete spherical surface. T'he average value of $\sin ^{2} \theta$ is $\frac{2}{3}$, and hence the plane-wave energy, reckoned in one direction only, bears to the scattered energy the ratio

$$
\text { [Type I.] } 3 \sigma \lambda^{2} / 8 \pi=3 \pi \sigma / 2 v^{2} . . . . . .
$$

42. A radiator of type II. has an axis fixed in it, with respect to which its vibrations are understood to be always symmetrical. Let such a radiator be exposed to the action of a primary plane-polarized disturbance, with electric vector parallel to the $z$-axis, the angle $\phi$ between the axis of the radiator and the $z$-axis being in general finite. The amplitude of vibration will be only $\cos \phi$ times as great as if the two directions had agreed; and we can, moreover, resolve the secondary disturbance into two components : one with electric vector parallel to the plane of $x y$, the other with electric vector parallel to the axis of $z$. The amplitude of this latter component is only $\cos ^{2} \phi$ times as great as if $\phi$ had been zero. Let the problem of $\S 41$ be now modified by substituting secondary radiators of type II. for those of type $I$., the axes of the radiators being oriented indiscriminately; and suppose that plane-polarized plane-waves such as those represented by (4.5) are incident upon the sheet of secondary radiators. Then evidently the only regular waves 
emitted are plane-polarized, with electric vector parallel to $z$; all else belongs to the irregular disturbance. If $a$ now represents the amplitude at distance $f$ due to a single secondary radiator, when the axis of the radiator is parallel to the $z$-axis, and the distance $f$ perpendicular thereto, it is evident that the resultant amplitude at any point of the diffraction pattern contains as a new factor the average value of $\cos ^{2} \phi$, where $\phi$ may be regarded as the co-latitude of any point on the surface of a sphere. The average of $\cos ^{2} \phi$ is $\frac{1}{3}$, so that at each point of the diffraction pattern the amplitude of disturbance is $\frac{1}{3}$ as great and the energy-flux $\frac{1}{9}$ as great as if the secondary radiators had been of type I. At the same time the expression for the scattered energy contains a new factor av. $\cos ^{2} \phi$ or $\frac{1}{3}$; and accordingly the regularly reflected energy bears to the scattered energy a ratio one-third as great as that given by $(50)$, namely,

[Type II.]

$$
\sigma \lambda^{2} / \measuredangle \pi \text { or } \pi \sigma / 2 v^{2} \text {. }
$$

43. Now let the whole plane of $y z$ be scattered over with secondary radiators, whether of type I. or of type II. ; the distribution (of $\sigma$ radiators per unit of area) being completely irregular, while the average vector-potential due to a single radiator is given, as regards direction and magnitude, by

$$
\mathbf{a}_{n}^{\prime}=0,0,{ }^{\prime} r_{n}^{-1} \exp i\left(p t-v r_{n}-\gamma\right) . \quad \text {. . }
$$

Then the vector-potential in the plane-waves propagated in the direction of $x$ increasing will be

$$
\mathbf{a}^{\prime \prime}=0,0, \Sigma \mathrm{C} r_{n}^{-1} \exp i\left(p t-v r_{n}-\gamma\right) \text {. }
$$

As in the diagram in Part I., let $O$ be the origin, $P$ a point $(x, 0,0)$ and $\rho^{2}=y^{2}+z^{2}$. If $\mathrm{Q}$ is any point in the $y z-$ plane, distant $\rho$ from the origin and $s$ from the point $\mathrm{P}$, the angle $\epsilon$ made by $P Q$ with the axis of $z$ is given by

$$
\cos \epsilon=\sin \theta \cos \phi \text {; }
$$

where $\theta$ is the angle $O P Q$, and $\phi$ the angle made by $O Q$ with the axis of $z$. For the moment we are concerned only with the regular waves, which depend on average values, and not with the diffuse radiation, which depends on deviations from the average: thus from symmetry the resultant vector potential at any point is always parallel to the $z$-axis. The contribution of an elementary area in the neighbourhood of $Q$ has to be twice resolved through an angle $\frac{\pi}{2}-\epsilon$, so that the corresponding element of the vector-potential at $\mathrm{P}$ 
will contain a factor $\sin ^{2} \epsilon=1-\sin ^{2} \theta \cos ^{2} \phi$; the average value of which, for any given value of $\theta$, is

$$
1-\frac{1}{2} \sin ^{2} \theta=\frac{1}{2}\left(1+\frac{x^{2}}{s^{2}}\right) \text {. }
$$

Thus to the $z$-component of the vector-potential at $\mathrm{P}$ the annulus $2 \pi \rho d \rho$ (or $2 \pi s d s$ ) contributes

$$
\mathrm{C} s^{-1} \exp i(p t-v s-\gamma) \cdot \sigma \cdot 2 \pi s d s \cdot \frac{1}{2}\left(1+\frac{x^{2}}{s^{2}}\right),
$$

the integral of which is

$$
\pi \sigma \mathrm{C} \int_{0}^{\mathrm{R}} \exp i(p t-v s-\gamma)\left(1+\frac{x^{2}}{s^{2}}\right) d s . .
$$

As in $\S 11$, the limits of integration are $s=x$ and $s=\mathrm{R}$, where $\mathrm{R}$ is very great compared with $x$.

44. The first term in (53) is (within a constant term)

$$
\begin{aligned}
& -i \pi \sigma \mathrm{C} v^{-1} \exp i(p t-v x-\gamma) \\
& \quad=-\pi \sigma \mathrm{C} v^{-1} \exp i\left(p t-v x-\gamma+\frac{1}{2} \pi\right) .
\end{aligned}
$$

The second term in (53) depends on the integration of $s^{-2} \exp (-i v s) d s$ between the limits $s=x$ and $s=\mathrm{R}$. Its value is readily found by successive integration by parts, if we remember that $v x$ is large and may be chosen as large as we please, provided only that $\mathrm{R}: x$ is very large. The value finally obtained for (53) is

$$
-2 \pi \sigma \mathrm{C}^{-1} \exp i\left(p t-v x-\gamma+\frac{1}{2} \pi\right),
$$

and the waves propagated in either sense from the plane of $y \approx$ are given by

$$
\mathbf{a}^{\prime \prime} ; \mathbf{a}^{\prime \prime \prime}=0,0,-2 \pi \sigma \mathrm{C} v^{-1} \exp i\left(p t \mp v x-\gamma+\frac{1}{2} \pi\right) .
$$

45. Suppose now that the secondary radiators, irregularly distributed in the plane of $y z$, are sending forth disturbances (represented on an average by (52)) owing to the incidence of primary waves

$$
\mathbf{a}=0,0, \mathrm{~A} \exp i(p t-v x) ; . . . .
$$

and let $\sigma \lambda^{2}$ be so small that each radiator is sensibly uninfluenced by the disturbances reaching it from its neighbours. If the radiators are of type I. we can apply (49); $\gamma$ being the phase-lag in the case of an isolated secondary radiator. The secondary plane waves are thus [Type I.]

$\mathbf{a}^{\prime \prime} ; \mathbf{a}^{\prime \prime \prime}=0,0,-3 \pi \sigma A v^{-2} \sin \gamma \exp i\left(p t \mp v x-\gamma+\frac{1}{2} \pi\right)$. 
If we are dealing (as in $\$ 22$ ) with a thin lamina $0<x<\delta x$, with $v$ secondary vibrators of type I. per unit of volume, $\sigma$ in (56) must be replaced by $v \delta_{i} v$, and corresponding to the resultant incident waves (55), the secondary waves emitted are

where

$$
0,0,-\chi \delta x \cdot A \exp i(p t \mp v x),
$$

$$
\text { [Type I.] } x=3 \pi \nu \nu^{-2} \sin \gamma \exp i\left(\frac{1}{2} \pi-\gamma\right) .
$$

46. Under the primary stimulus (55), when the secondary radiators are of type II., with axes promiscuously oriented, the average radiator gives out a disturbance which differs from that for a type 1 . radiator only in having an additional factor $\frac{1}{3}$, as explained in $\S 42$. Thus

$$
\text { [Type II.] } \chi=\pi \nu \boldsymbol{v}^{-2} \sin \gamma \exp i\left(\frac{1}{2} \pi-\gamma\right) \text {. }
$$

47. Either $(57 a)$ or $(57 b)$-according to the type of secondary radiator with which we have to deal-may now be made use of in adapting some results already obtained to the circumstances of optical problems. For instance, equations (9) and (24) still hold good, with the single emendation that the value attributed to $w$ is multipled by $\frac{2}{2}$ or by $\frac{1}{2}$ as the case may be. In particular, when the radiators are tuned as resonators, $w=3 \pi \sigma v^{-2}$ (Type I.) or $v=\pi \sigma v^{-2}$ (Type II.), and as before the validity of (9) and (24) is limited only by the conditions that the radiators must seatter without absorbing wave-energy of frequency $p / 2 \pi$, and must be distributed with perfect irregnlarity like gas-molecules. The expressions (50), (51) likewise hold good rigoronsly provided the same conditions are fulfilled. It appears from Wood's researches that, as the density of a swarm of resonant molecules is increased, the condition that true absorption should be absent fails long before any marked departure from the law's of ideal gases has become apparent. This is so even in the case of mercury vapour, which can be raised to a pressure of several atmospheres without destroying the property of resonance.

48. Equation (36), giving the amplitude of waves regularly reflected from an attenuated swarm of secondary vibrators, must now be replaced by

$$
\left.\begin{array}{ll}
\text { [Type I.] } & \mathrm{A}^{\prime \prime \prime}=-\frac{3}{2} \mathrm{~A} i \pi \nu v^{-3}=-3 \mathrm{~A} i \nu \lambda^{3} / 16 \pi^{2} \\
\text { [Type II.] } & \mathrm{A}^{\prime \prime \prime}=-\frac{1}{2} \mathrm{~A} i \pi \nu v^{-3}=-\mathrm{A} i \nu \lambda^{3} / 16 \pi^{2}
\end{array}\right\} .
$$

Phil. Mag. S. 6. Vol. 30. No. 175. July 1915. H 
Again, in place of (40) we shall have

$\left.\begin{array}{ll}\text { [Type I.] } & \chi_{2} v^{-1}=\frac{3}{2} \pi \nu v^{-3} \sin 2 \gamma=3 \nu \lambda^{3} \sin 2 \gamma / 16 \pi^{2} \\ \text { [Type II.] } & \chi_{2} v^{-1}=\frac{1}{2} \pi \nu v^{-3} \sin 2 \gamma=\nu \lambda^{3} \sin 2 \gamma / 16 \pi^{2}\end{array}\right\} ;$

and in place of (41), for the extinction-coefficient,

$\left.\begin{array}{ll}\text { [Type I.] } & \chi_{1}=3 \pi \nu v^{-2} \sin ^{2} \gamma=3 \lambda^{2} \nu \sin ^{2} \gamma / 4 \pi \\ \text { [Type II.] } & \chi_{1}=\pi \nu v^{-2} \sin ^{2} \gamma=\lambda^{2} \nu \sin ^{2} \gamma / 4 \pi\end{array}\right\}$.

49. The two types of molecule which have bean especially considered, though far from exhausting the a priori possibilities, may serve as examples ; and it sbould be possible to decide experimentally whether type I. or type II. represents the more nearly the properties of the actual molecules of any given gas-or whether a type distinctly different from either is indicated. The suggestions now offered in this connexion relate only to the means of discriminating between molecules of type I. and those of type II. The most obvious method is to use piane-waves completely polarized as the primary disturbance, and to observe the intensity and degree of polarization of the diffuse secondary radiation emitted in definite directions. The density of the vapour should in these tests be small enough to make the tertiary etc. radiation insignificant in comparison with the secondary; otherwise the distinctions to be observed would be partially obliterated, and the whole problem would become more complicated. From the fact that Wood * observad no trace of polarization in the radiation emitted laterally by his "resonance lamp," it may be surmised that a considerable proportion of the resonance radiation was of tertiary or higher order. For it seems hardly possible to imagine a molecule such that the secondary $f$ radiation emitted perpendicularly to the original existing beam would not be at least partially polarized. Indeed Wood, in the papers referred to, has emphasized the prominence of the radiation of higher orders. To secure an approximately pure secondary radiation, it may bo necessary to use mercury vapour under as low a pressure as $.001 \mathrm{~mm}$. contained in a vessel of much smaller dimensions than those bitherto used. In the following two paragraphs it is assumed that such precautions have been taken.

* Phil. Mag. May 1912, p. 712.

$\dagger$ What is referred to above as "secondary radiation" is the same as Wood's "primary resonance radiation," the term "primary" being in this paper applied to the original incident benm. 
50. As hitherto, let the wave-fronts of the primary disturbance be parallel to the plane of $y z$, with the electric vector parallel to the $z$-axis; and let the resonant gasmolecules be contained in a rectangular vessel with transparent sides parallel to the three co-ordinate planes. Then, if the molecules are of type I., each of them will be vibrating symmetrically with respect to an axis parallel to the $\mathrm{ixis}$ of $z$, and the following deductions can be immediately made. The secondary diffuse radiation proceeding in any assigned direction will be fully polarized, and in a direction making an angle $\theta$ with the axis of $z$, the intensity of the secondary diffase radiation will be proportional to $\sin ^{2} \theta$.

51. Alternatively, suppose the molecules to be of type II., each having fixed within it a definite axis, with respect to which any induced vibration will be symmetrical. Consider a molecule whose axis is inclined $\theta$ to the $z$-axis, while the plane parallel to the $z$-axis through the axis of the molecule makes an angle $\phi$ with the plane of $x z$. Then the vibration of the molecule can be resolved into three rectangular components having amplitudes proportional to

$$
\sin \theta \cos \phi, \quad \sin \theta \sin \phi, \quad \cos \theta .
$$

For diffuse secondary radiation emitted in the direction of the axis of $x$, the polarized components have intensities in the proportion

$$
\text { av } \cos ^{2} \theta: \operatorname{av} \sin ^{2} \theta \sin ^{2} \phi=2: 1
$$

averag ${ }^{2}$ values being denoted by the prefix av, and the stronger component being that for which the electris vector is parallel to the axis of $z$. The same evidently holds good for the diffuse secondary radiation emitted in any direction parallel to the plane of $x y$. For a direction parallel to the axis of $z$, the ratio is one of equality, the radiation being unpolarized. Under the conditions contemplated in this paragraph, it is easily seen that the total intensity of diffuse secondary radiation emitted parallel to the axis of $y$ bears to that emitted parallel to the axis of $z$ the ratio $3: 2$.

52. From his experimental study of the absorption of radiation $(\lambda 2536)$ in mercury-vapour at low pressure, with some considerations of a general nature, Wood has drawn the conclusion that, at any given instant, only a small proportion of the mercury molecules are acting as resonators. This seems to be borne out by a somewhat more detailed examination of the question. In Wood's experiments the radiation from a quartz mercury arc, restricted to $\lambda 2536$, H 2 
was caused to pass through mercury vapour at a pressure of $.001 \mathrm{~mm}$. The radiation laterally scattered was most intense where the primary beam entered, and gradually fell off as the beam penetrated further into the vapour; the intensity being reduced to one-half after a depth of $5 \mathrm{~mm}$. had been traversed. 'The proportional reduction of amplitude in traversing a layer comparable in thickness with $\lambda / 2 \pi$ is evidently quite small, and the approximations of $\S 34$ can be used. For the present let attention be confined to the primary (or incident) and secondary radiations; also let the possible influence of the Doppler effect and of collisions between molecules be disregarded.

53. The coefficient of extinction, for light of any assigned frequency, is proportional to $\sin ^{2} \gamma$, where $\gamma$ is the amount by which the secondary radiation from a molecule lags in phase behind the resultant incident radiation: a result derived from the sole assumption that no absorption of energy takes place. But when we want to express $\gamma$ as a function of the particular frequency $(p)$ in question, some further assumption must be made, and one that readily presents itself is that $\gamma$ is related to $p$ as it would be in a purely dynamical system. To express such a relation, new quantities have to be introduced, though these do not appear in the final result. If the free vibrations of a singly-free system with co-ordinate $u$ are conditioned by $y^{*}$

$$
\ddot{u}+\kappa \dot{u}+n^{2} u=0,
$$

so that the natural (undamped) frequency is $n / 2 \pi$, then the prolonged action of a force proportional to $\cos p t$ will give rise to a vibration which lags in phase behind the force by $\gamma$, where

$$
\tan \gamma=p \kappa /\left(n^{2}-p^{2}\right) \dagger .
$$

Since the effective range of frequency with which we are. concerned is extremely narrow, no appreciable error is involved in replacing this last equation by

$$
\tan \gamma=n \kappa /\left(n^{2}-p^{2}\right)
$$

whence

$$
\sin ^{2} \gamma=\frac{n^{2} \kappa^{2}}{\left(n^{2}-p^{2}\right)^{2}+n^{2} \kappa^{2}} \cdot \quad . \quad . \quad .
$$

54. Let the energy-flux in the incident beam between the limits $p^{2}=p^{2}$ and $p^{2}=p^{2}+d\left(p^{2}\right)$ be $f^{\prime}(p) d\left(p^{2}\right)$. Then since

* In the present optical application, the presence of the term $к \dot{i}$ must be attributed wholly to radiation from the molecule.

+ Rayleigh, 'Theory of Sound,' vol. i. $\$ 46$. 
the values of $p$ which are effective belong to a very narrow spectrum-line in the middle of a much broader incident line, we may simplify by treating $f(p)$ as a constant. After a thickness ( $L$ say) of the vapour has been traversed, the distribution of energy in the primary beam will be different. For any assigned value of $p$, the energy of the primary beam has been changed in the proportion $\exp \left(-\chi_{1} \mathrm{~L}\right)$, and $\chi_{1}$ as we know is proportional to $\sin ^{2} \gamma$; so that in place of $\exp \left(-\chi_{1} L\right)$ we may put $\exp \left(-K \sin ^{2} \chi\right)$, where $K$ is a constant multiple of $L$, and is equal to $\chi_{1} L \operatorname{cosec}^{2} \gamma$. At the same time it has to be remembered that, corresponding to any definite value of $p$ (which determines $\gamma$ ), the diffuse secondary radiation emitted per molecule * has an intensity proportional jointly to the intensity of the exciting radiation and to $\sin ^{2} \gamma$. It follows that the intensity of the diffuse secondary radiation at a depth $\mathrm{T}$, within the mass of vapour will be less than that where the primary beam first enters in the ratio

$$
\int \sin ^{2} \gamma \cdot \exp \left(-\mathrm{K} \sin ^{2} \gamma\right) d\left(p^{2}\right): \int \sin ^{2} \gamma d\left(p^{2}\right) .
$$

Making use of (61), and at the same time putting $M$ in place of $\left(p^{2}-n^{2}\right) / n \kappa$, we can write for the ratio

$$
\int \frac{1}{\Pi^{2}+1} \exp \left(-\frac{\mathrm{K}}{\mathrm{M}^{2}+1}\right) d \mathrm{M}: \int \frac{1}{\mathrm{M}^{2}+1} d \mathrm{M} \text {. }
$$

Only a very suall range of values (negative and positive) of $M$ contributes sensibly to the integrals, and the limits of integration must be wide enough to include the whole of this range.

55. Using a rough graphic method, and ranging from $M=-10$ to $M=+10$ (or from 0 to 10 , which amounts to the same thing), I find that the ratio (62) would be as small as 0.5 probably for $K=1.5$ and certainly for $K=1 \cdot 6$. The latter figure may be compared with Wood's experimental result, referred to in $\$ 52$ above. Thus

$$
1 \cdot 6=K=\chi_{1} \operatorname{cosec}^{2} \gamma \times 0.5 \mathrm{~cm}
$$

On reference to $(60)$ the values to be attributed to $\nu$ (the number of resonators per c.em.) are seen to be

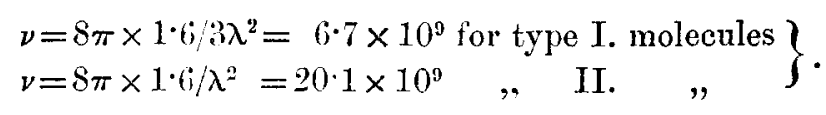

* The energies of the diffuse secondary radiations from the various molecules being simply additive in an attenuated vapour sensibly obeying the gas-laws ( $f . \& 7$, part I.). 
The actual density of mercury vapour was equivalent to one molecule in a volume $\lambda^{3}$, or $6^{\circ} 07 \times 10^{13}$ molecules per c.c.; and thus (63) implies that at any instant only about one in 9000 (one in 3000) of them are effective resonators: the molecules being of type I. (type II.). This agrees with Wood's qualitative conclusion.

56. There are, however, some further considerations which should not be lost sight of, and which seem to indicate that one in 9000 (or one in 3000) is an under-estimate. In the first place the secondary diffuse radiation, though effectively restricted to an extremely narrow range of frequency, is far from homogeneous in regard to its absorbability by mereury vapour: and that which is excited well within the mass of vapour is due to a primary beam in which the more absorbable frequencies have already been selectively weakened. It is thus less absorbable than that excited where the primary beam first enters. Since the scattered radiation has to traverse some thickness of mercury vapour before escaping through the quartz window at the side of the vessel, it follows that the diffuse brightness along the track of the primary beam will appear more uniform than it should, thus leading to an under-estimate of the primary absorption. At the same time the deviation of the general course of absorption from a simple exponential law will be rendered less conspicuous; though Wood has already suspected from his measurements that such deviation exists.

57. In the next place, the energy removed from the primary beam takes the form of secondary radiations which in turn are found to excite tertiary radiations and so on; and thus along the course of the primary beam the falling off in the total radiation available for exciting the resonant molecules is less rapid than the extinction of the primary beam itself.

58. Incidentally it should be remarked that, when the highest homogeneousness in the radiation from a resonance lamp is desired, the total thickness of vapour traversed by incident and scattered radiation should be very moderate; the scattered energy being then necessarily but a small part of the incident, even in the case of the most effective frequencies.

59. It is not easy to make satisfactory allowance for the sources of error referred to in $\$ \S 56,57$. From the point of view of theoretical treatment the following slightly modified method seems to have some advantages. A resonance lamp, fulfilling the condition referred to in the last paragraph, is used as a source of radiation, the illuminated object being a small 
hole in an opaque screen placed before the lamp. By means of. a quartz lens an image of the hole is formed on a photographic plate, a parallel-sided quartz cell, successively empty and filled with low-pressure mercury vapour, being interposed close to the lens. The illuminated hole being small, the image formed on the plate need be but little affected by the diffuse radiation from the absorbing cell. Since the radiation from the resonance lamp over the range of Irequency $p^{2}=x^{2}$ to $p^{2}=p^{2}+d\left(p^{2}\right)$ will be approximately proportional to $\sin ^{2} \gamma$, it is easy to see that the mercurial absorption will reduce the brightness of the image in the ratio (62); whence by arithmetical trial and error we can find $K$, that is $\chi_{1} \operatorname{cosec}^{2} \gamma \mathrm{L}$ (where $\mathrm{L}$ is the internal thickness of the cell), proceeding as in $\$ 55$.

60. From the form of the expression (62) it seems to be a not unreasonable conclusion that, when resonance radiation, due to a single resonant frequency, is emitted from an attennuated vapour, the law of general enfeeblement of that radiation, when passing through the same vapour, depends only on the type and number per cubic centimetre of molecular resonators. It is here to be understood that the resonance radiation is emitted without either absorption or change of frequency, and that the original excitation is due to a "line" much wider spectroscopically than that which represents the resonance radiation. The suggestion is put forward with some reserve, because the relation (61) between $2 \pi \times$ frequency $(p)$ and phase-lag $(\gamma)$, involving also the dissipative coefficient $\kappa$, is derived from the assumption of a formal analogy between the resonating molecule and an ordinary dynamical system. However, $\kappa$ disappears from (62), and it is not easy to imagine the relation between frequency and phase-lag so different in form from (61) that the ratio (62) would have to be replaced by one of a difterent order of magnitude. The conclusion that only one molecnle of mercury vapour in several thousand forms an effective resonator for $\lambda 2536$ suggests the question : to how many classes, differing spectroscopically from one another, do the mercury molecules belong? And in a vapour such as that of iodine, which yields very complicated resonance spectra, is the number of spectroscopic classes greater? Determinations of the extinction of iodine-resonance-radiations by iodine vapour, under conditions precluding true absorption, might help to indicate how many effective resonators are present for each line examined; but here fresh complications arise.

61. In the foregoing discussions, the aim has been to 


\section{Scattering and Reflexion of Light by Gas Molecules.}

assume as little as possible regarding the processes of radiation; the activity of a radiating molecule has been specified merely by the disturbance produced at a distance, and it may be remarked that the validity of the results is not directly conditioned by the smallness of the molecules against the wave-length involved; but it has been frequently assumed that the molecules are distributed approximately as in an ideal gas, and thus many of the formula would fail if the linear dimensions of the molecules were comparable with their mean free path. The influence of the Doppler effect and of collisions between molecules must be very small in pure mercury vapour at room temperature; this is indicated by elementary gas-theory, and is confirmed by the experimental evidence for the extreme narrowness of the resonance line 2536.

62. More doubt may be felt as to the assumed absence of true absorption in the pure attenuated vapour. The assumption is supported on general grounds by Wood, who insists, however, on the urgent need for verification. And here I venture to suggest that, provided sufficient sensitiveness can be realized, a satisfactory way of testing for absorption of radiation is to determine the value (whether zero or finite) of some effect to which absorption would directly give rise. Absorbed radiation will, in fact, heat the vapour and any air that may be mixed with it, thus causing expansion ; and there seems to be reason for supposing that the sensitiveness obtainable on these lines would be abundant for the purpose in view. The form of apparatus proposed is indicated in plan in the diagram; it was suggested by F. W. Jordan's

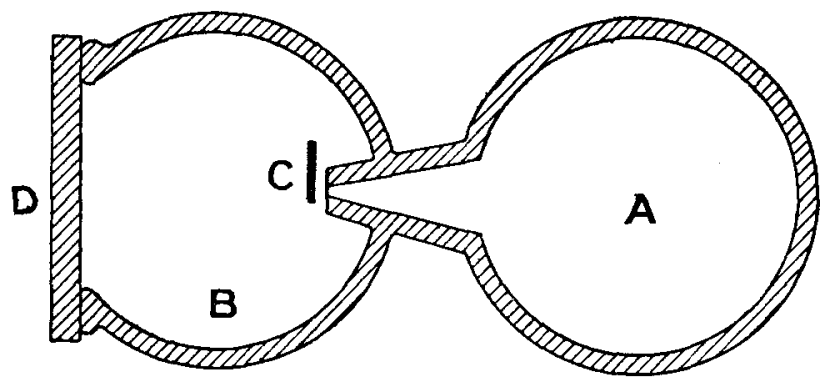

paper * "On a new type of thermo-galvanometer." A is a little flask of fused silica, whose neck projects into, and is fused to, the bulb $B$. $C$ is a small thin circular disk, preferably of very thin fused silica, platinized. This is suspended

* Phys. Soc. Proc. vol. xxvi. part iii. (A pril 1914). 
Number of Flectrons concerned in Metallic Conduction. 105

in the position indicated by a fine quartz fibre, the bulb $B$ being provided with a vertical tube (not shown) for carrying the suspension. If we begin leating the residual air or vapour in $A$, a puff will issue from the neck, and will turn the vane $C$ in azimuth, the deflexion being read through the window D. A good notion of suitable dimensions can he gained from Jordan's paper. Meanwhile it may be mentioned that one of that author's galvanometers gave a deflexion of $4 \mathrm{~mm}$. at a scale-distance of $64 \mathrm{~cm}$. for a steady heating of one micro-watt suddenly commenced; the maximum excursion being reached in about 2 seconds, after which the vane gradually recovered its normal position. In this case the air was at atmospheric pressure, and general considerations seem to suggest that at lower pressure a greater effect should be obtained for a like rate of heat-production.

63. Suppose that, to begin with, the system contains air at $01 \mathrm{~mm}$. pressure ${ }^{*}$, free from mercury vapour. A beam from the resonance-lamp is suddenly made to fall on $\mathbf{A}$, and if the vano $C$ executes a significant movement, the extent of this is noted. (Probably the movement of the rane would be very small, for in 2 seconds or so any slight absorption by the silica walls would hardly have time to affect the temperature of the contained air perceptibly.) A drop of mercury is now introduced, and the test repeated; in the absence of true absorption the excursion of the vane should be about the same as before. The apparatus would of course need calibrating, if only roughly. Further details would be out of place here, but I cannot help thinking that in some such manner definite results might be obtained by a slilful observer.

Boar's Hill, Oxford, 3rd May, 1915 .

\section{On the Number of Electrons concerned in Metallic Conduction. By G. H. Livens †.}

THE mathenatical theory of metallic conduction has been 1 extensively developed by various writers wilh a view not only to placing the theory on as firm a foundation as possible, but also with the object of determining more precisely the general electrical properties of the metals. The results of certain forms of theory are certainly in fair agreement with experiment, but the agreement obtained, even

* This air-pressure is suggested because Vood has found it to have no perceptible influence on the absorption of $2536^{\circ}$ radiation by mercuryrapour at room temperature.

+ Communicated by the Anthor. 